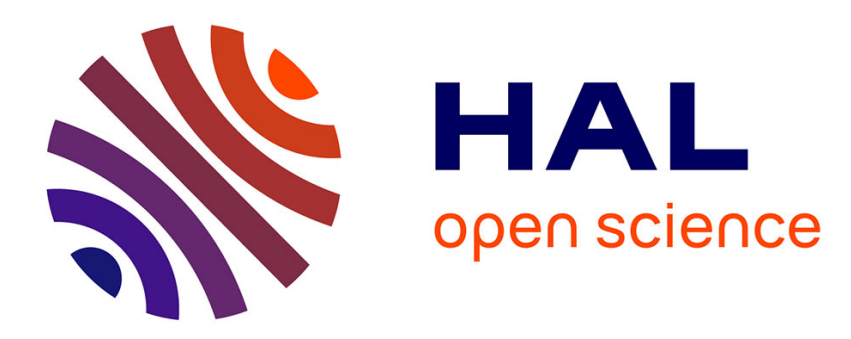

\title{
La géographie industrielle, discipline en crise dans la recherche russe
}

Denis Eckert, Andreï Treïvich

\section{To cite this version:}

Denis Eckert, Andreï Treïvich. La géographie industrielle, discipline en crise dans la recherche russe.

Espace Géographique, 1995, 10.3406/spgeo.1995.3371 . halshs-02565752

\section{HAL Id: halshs-02565752 \\ https://shs.hal.science/halshs-02565752}

Submitted on 6 May 2020

HAL is a multi-disciplinary open access archive for the deposit and dissemination of scientific research documents, whether they are published or not. The documents may come from teaching and research institutions in France or abroad, or from public or private research centers.
L'archive ouverte pluridisciplinaire HAL, est destinée au dépôt et à la diffusion de documents scientifiques de niveau recherche, publiés ou non, émanant des établissements d'enseignement et de recherche français ou étrangers, des laboratoires publics ou privés. 


\title{
La géographie industrielle, discipline en crise dans la recherche
}

\section{russe}

\section{Denis Eckert, Andrei Treïvich}

\section{Résumé}

La géographie industrielle en Russie est en proie à un déclin, ce dont témoigne le tarissement des publications à partir de 1970 . Cette crise se manifeste au moment même où l'ampleur des restructurations du tissu productif appellerait un renouvellement complet des études et des concepts, comme un investissement des chercheurs.

\begin{abstract}
Industrial geography, a discipline in crisis in Russian research. - Industrial geography in Russia has fallen into decline, as evidenced by the drying up of publications after 1970. This crisis has manifested itself at the very time when large-scale restructuring of the productive fabric ought to call for a thorough renewal of studies and concepts and investment on the part of researchers.
\end{abstract}

\section{Citer ce document / Cite this document :}

Eckert Denis, Treïvich Andrei. La géographie industrielle, discipline en crise dans la recherche russe. In: Espace géographique, tome $24, n^{\circ} 2,1995$. pp. 169-180;

doi : https://doi.org/10.3406/spgeo.1995.3371

https://www.persee.fr/doc/spgeo_0046-2497_1995_num_24_2_3371

Fichier pdf généré le 03/01/2019 


\title{
Les champs de la géographie
}

\section{La géographie industrielle, discipline en crise dans la recherche russe}

\author{
Denis Eckert \\ Chargé de recherche au CNRS \\ Maison de la Géographie, 17 rue Abbé de l'Épée 34000 Montpellier
}

\author{
Andreï Treivich \\ Chercheur à I'Institut de géographie de l'Académie des sciences de Russie \\ Staromonetny per. 29, 109017 Moscou
}

RÉSUMÉ.- La géographie industrielle en Russie est en proie à un déclin, ce dont témoigne le tarissement des publications à partir de 1970. Cette crise se manifeste au moment même où l'ampleur des restructurations du tissu productif appellerait un renouvellement complet des études et des concepts, comme un investissement des chercheurs.

BIBLIOMÉTRIE, GÉOGRAPHIE INDUSTRIELLE, RUSSIE
ABSTRACT. - Industrial geography, a discipline in crisis in Russian research.- Industrial geography in Russia has fallen into decline, as evidenced by the drying up of publications after 1970. This crisis has manifested itself at the very time when large-scale restructuring of the productive fabric ought to call for a thorough renewal of studies and concepts and investment on the part of researchers.

BIBLIOMETRY, INDUSTRIAL GEOGRAPHY, RUSSIA
La géographie industrielle fut un temps la branche la plus importante et la plus valorisée de la géographie humaine soviétique, notamment quand ses représentants collaboraient activement aux grands projets d'aménagement et de mise en valeur du territoire. Aujourd'hui, cette discipline est en grave crise: ses modèles de référence ont disparu et, dans les incertitudes actuelles, aucune école n'émerge en prenant en main l'analyse de la transition industrielle.
Cette crise de la géographie industrielle en Russie est un cas spécifique: à l'intérieur de la géographie humaine, la géographie politique, sociale, les études urbaines, etc., ont au contraire profité de la fin du système communiste. Elles se sont renouvelées et font preuve, malgré la situation critique de la recherche, d'un grand dynamisme (Kolossov, 1993). Même la géographie physique, si hégémonique et naturaliste naguère, a su s'intéresser aux 
enjeux de la gestion de l'environnement, prendre en compte la notion de risque tout en renouvelant ses approches instrumentales (Intercarto, 1994).

Nous voudrions montrer que cette crise de la géographie industrielle, qu'on pourrait être tenté d'expliquer seulement par le changement brutal et récent de système économique, s'enracine dans le passé soviétique proche et que les prémisses du déclin étaient déjà perceptibles il y a dix ou vingt ans. Ce phénomène s'inscrit d'ailleurs dans un mouvement général de déclin de la géographie industrielle dans le Monde.

Pour mieux le mesurer, nous avons eu recours à la bibliométrie, afin d'avoir une vision aussi étendue que possible de la place de la géographie industrielle dans les principales revues scientifiques, et pour pouvoir juger, d'un point de vue quantitatif, son évolution par rapport aux autres grands domaines de recherche. Ce que l'on perd ainsi en contenu, en renonçant à analyser les articles eux-mêmes et leurs problématiques, on le gagne en exhaustivité.

\section{La crise après «l'âge d'or»?}

La faiblesse actuelle de la géographie industrielle en Russie est d'autant mieux perçue qu'elle contraste avec un passé où elle tenait le haut du pavé, ce qui pourrait porter, chez les spécialistes, à créer la nostalgie d'un «âge d'or». L'enjeu de l'industrialisation de l'ancienne Union soviétique a en effet porté l'essor de la géographie industrielle pendant plusieurs décennies. De très nombreux chercheurs ont, dès la fin de la guerre civile, apporté leur contribution à l'étude des phénomènes de croissance, d'aménagement et de planification industriels dans l'espace soviétique.

Dès les années 1920 avec N. Baranski, l'école de géographie industrielle de l'université de Moscou a été la plus active. Après la seconde guerre mondiale, l'un de ses représentants, N. Kolossovski, a formulé les notions de complexe territorial de production et de cycle productif que l'on peut rapprocher de la théorie des pôles de croissance de François Perroux. L'objectif était, pour Kolossovski et son groupe, d'élaborer un ensemble d'outils pouvant servir le développement régional global, tous secteurs inclus. De fait, ces outils furent surtout appliqués, en théorie comme dans la pratique, à la question du développement industriel, surtout dans le secteur minier et dans l'industrie lourde.

Après la période de gloire (1950-1960) de cette géographie volontariste, tournée vers l'aménagement et franchement positiviste, on assiste à l'émergence d'approches critiques: les articles de A. Probst, N. Alisov, V. Gorlov, I. Mayergoiz ou N. Arianine en témoignent dès le milieu des années 1960. On y montre l'immixtion croissante des ministères centraux dans la gestion du développement régional, la tendance au ralentissement du développement industriel, les inégalités spatiales de la croissance. Mais ces auteurs notent aussi que l'industrie a gagné en autonomie dans ses décisions de localisation, la pression des autorités centrales s'étant quelque peu relâchée sur ce point, laissant ainsi un espace de liberté aux directeurs d'entreprise.

De nouveaux champs d'analyse et d'observation des dynamiques industrielles semblent alors s'ouvrir. Les quelques tentatives théoriques faites pour renouveler les bases conceptuelles de la géographie industrielle sont certes plutôt timides mais, dans les années 1970, l'optimisme est toujours de mise: les chercheurs pensent pouvoir faire progresser leur discipline... Et croient aussi au développement industriel de l'URSS. Tout cela est aujourd'hui de l'histoire ancienne. Quelque part entre le début des années 1970 et les années 1980, le déclin de la géographie industrielle commence, perceptible dans la place que lui réservent les principales revues scientifiques.

\section{Le déclin de la géographie industrielle dans les revues scientifiques}

\section{Méthode d'analyse des publications}

Nous avons basé l'analyse (1) qui suit sur le dépouillement de quatre revues: Izvestia Akademii Naouk, seria geografitcheskaïa (IAN dans la suite de cet article), Moscou (Bulletin de l'Académie des sciences); Viestnik Moskovskogo Universitieta (VMU), Moscou (Courrier de l'université de

(1) En reprenant et complétant, pour les années 1990, le travail déjà effectué par $\mathrm{A}$. Treïvich (1982). 
Moscou); Izvestia Vsiosoïouznogo (maintenant Rossiiskogo) Geografitcheskogo Obchtchestva (IVG0), Léningrad-SaintPétersbourg (Bulletin de la Société géographique de l'URSS - aujourd'hui Russe); Geografia i Prirodnye Resoursy (GPR), Irkoutsk (2) (Géographie et ressources naturelles). Cet échantillon correspond aux revues qui ont la meilleure notoriété. IAN, revue publiée par l'Académie des sciences, est la plus connue. $V M U$ est la revue de la plus grande faculté de géographie du pays, comptant le plus grand nombre d'étudiants, d'enseignants et de chercheurs. IVGO est la plus ancienne revue de géographie du pays: elle a été fondée au XIX ${ }^{\mathrm{e}}$ siècle par la Société de géographie impériale. GPR est certes la revue la plus récente et la plus "provinciale» (elle est publiée à Irkoutsk), mais elle a su gagner un certain rayonnement, tout en restant très spécialisée dans les domaines de l'environnement et de la mise en valeur des ressources naturelles, avec des articles plutôt orientés vers les études de cas et la géographie appliquée.

Ces quatre revues rassemblent l'essentiel des contributions de géographie humaine. Ce sont par ailleurs celles qui ont la meilleure audience internationale: elles sont dépouillées en priorité dans les bibliographies (la Bibliographie géographique internationale éditée par le Centre national de la recherche scientifique français par exemple). Pour simplifier le travail de dépouillement, seules les trois premières années de chaque décennie ont été prises en compte, à savoir 1970-1971-1972, 1980-1981-1982, 1990-1991-1992. En raison de la grave crise de l'édition scientifique dans la Russie actuelle, à la date du $1^{\text {er }}$ avril 1993, aucune des quatre revues n'avait publié l'intégralité de ses numéros 1992 (VMU et GPR seulement deux, IVGO trois et IAN quatre). Les délais de publication des manuscrits (de un à deux ans dans l'ancien système académique) peuvent peutêtre aussi quelque peu fausser l'analyse, en introduisant un décalage artificiel entre l'émergence des tendances et leur traduction éditoriale.

(2) La recension effectuée dans les années 1980 par A. Treivich ne prenait pas en compte cette revue sibérienne, fondée en 1980 seulement; ce n'est qu'aujourd'hui que l'on peut apprécier l'évolution de son contenu avec suffisamment de recul.
Le nombre total d'articles pris en compte est de 3734 , soit 20 en moyenne par numéro de revue (3). Mille d'entre eux ont pu être identifiés comme relevant de la géographie humaine, économique ou sociale, qui seules nous intéressent. On a eu recours aux mots-clés pour classer les articles, sans analyse de leur contenu, pratiquement impossible compte tenu du nombre de publications prises en compte, et retenu huit genres permettant de classer les articles traitant de géographie humaine. Il est difficile dans la pratique d'éviter certaines approximations. La huitième et dernière catégorie de notre classification concerne l'étude des ressources naturelles et de l'environnement et a posé le problème de son inclusion dans la géographie humaine. Certains termes caractéristiques comme prirodopolzovanie (utilisation de la nature) ou retombées économiques de la pollution, etc., ont pu orienter notre choix et le nom de l'auteur a parfois pu nous aider à trancher.

Une fois ce problème résolu, on se retrouve face à la validité des limites entre les genres identifiés à l'intérieur de la géographie humaine, comme par exemple entre géographie économique «industrielle» et «générale». Ce dernier genre, qui regroupe environ $24 \%$ du total des articles socio-économiques recensés, correspond à une grande variété de sujets: aussi bien des articles théoriques, méthodologiques, que de synthèse régionale (kompleksnyi analiz), etc. Certains articles "généraux» des années 1970 (mots-clés: complexe territorial de production, structure territoriale de l'économie, ou encore razmechtchenie - localisation) renvoient, au moins en partie, à des faits de géographie industrielle. Néanmoins, l'absence de mots-clés spécifiquement «industriels» nous les a fait ranger dans la catégorie «générale».

\section{Un déclin précoce}

Les premiers signes de déclin de la géographie industrielle comme champ disciplinaire ont précédé le déclin industriel lui-même: la crise est sensible dès la fin des années 1970 .

(3) Il s'agit pratiquement de tous les articles, y compris les recensions d'ouvrages et les textes d'une page, à l'exception toutefois des hommages à des auteurs vivants ou décédés (jubilés, mélanges). 
Tabl. 1.-Analyse des mots-clés des principales revues.

\begin{tabular}{|c|c|c|c|c|c|c|c|c|c|c|}
\hline \multicolumn{11}{|c|}{ Nombre d'article pour les années 1970, 1971, 1972} \\
\hline \multirow{2}{*}{$\begin{array}{l}\text { Thèmes } \\
\text { Total }\end{array}$} & \multicolumn{2}{|c|}{ IAN } & \multicolumn{2}{|c|}{$V M U$} & \multicolumn{2}{|c|}{ IVGO } & & & \multicolumn{2}{|c|}{ TOTAL } \\
\hline & 410 & $\%$ & 449 & $\%$ & 348 & $\%$ & & & 1207 & $\%$ total \\
\hline Sous-total: géographie socio-économique & 106 & 25,9 & 106 & 23,6 & 56 & 16,1 & & & 268 & 22,2 \\
\hline 1 géographie socio-économique générale & 29 & 7,1 & 27 & 6,0 & 15 & 4,3 & & & 71 & 5,9 \\
\hline 2 géographie industrielle & 17 & 4,1 & 20 & 4,5 & 8 & 2,3 & & & 45 & 3,7 \\
\hline 3 autres productions matérielles & 7 & 1,7 & 8 & 1,8 & 3 & 0,9 & & & 18 & 1,5 \\
\hline 4 transport, commerce, services & 1 & 0,2 & 7 & 1,6 & 3 & 0,9 & & & 11 & 0,9 \\
\hline 5 population & 15 & 3,7 & 22 & 4,9 & 14 & 4,0 & & & 51 & 4,2 \\
\hline 6 géographie sociale, médicale, des loisirs & 3 & 0,7 & 4 & 0,9 & 0 & 0,0 & & & 7 & 0,6 \\
\hline 7 géographie politique, culturelle, ethnique & 1 & 0,2 & 0 & 0,0 & 4 & 1,1 & & & 5 & 0,4 \\
\hline 8 ressources naturelles et environnement & 33 & 8,0 & 18 & 4,0 & 9 & 2,6 & & & 60 & 5,0 \\
\hline \multicolumn{11}{|c|}{ Nombre d'articles pour les années 1980, 1981, 1982} \\
\hline Thèmes & \multicolumn{2}{|c|}{ IAN } & \multicolumn{2}{|c|}{$V M U$} & \multicolumn{2}{|c|}{ IVGO } & \multicolumn{2}{|c|}{$G P R$} & \multicolumn{2}{|c|}{ TOTAL } \\
\hline Total & 352 & $\%$ & 366 & $\%$ & 303 & $\%$ & 369 & $\%$ & 1390 & $\%$ total \\
\hline Sous-total: géographie socio-économique & 92 & 26,1 & 94 & 25,7 & 100 & 33,0 & 131 & 35,5 & 417 & 30,0 \\
\hline 1 géographie socio-économique générale & 18 & 5,1 & 26 & 7,1 & 33 & 10,9 & 15 & 4,1 & 92 & 6,6 \\
\hline 2 géographie industrielle & 6 & 1,7 & 17 & 4,6 & 16 & 5,3 & 25 & 6,8 & 64 & 4,6 \\
\hline 3 autres productions matérielles & 4 & 1,1 & 7 & 1,9 & 3 & 1,0 & 7 & 1,9 & 21 & 1,5 \\
\hline 4 transport, commerce, services & 4 & 1,1 & 7 & 1,9 & 5 & 1,7 & 6 & 1,6 & 22 & 1,6 \\
\hline 5 population & 22 & 6,3 & 9 & 2,5 & 25 & 8,3 & 10 & 2,7 & 66 & 4,7 \\
\hline 6 géographie sociale, médicale, des loisirs & 14 & 4,0 & 6 & 1,6 & 5 & 1,7 & 5 & 1,4 & 30 & 2,2 \\
\hline 7 géographie politique, culturelle, ethnique & 2 & 0,6 & 1 & 0,3 & 0 & 0,0 & 1 & 0,3 & 4 & 0,3 \\
\hline 8 ressources naturelles et environnement & 22 & 6,3 & 21 & 5,7 & 13 & 4,3 & 62 & 16,8 & 118 & 8,5 \\
\hline \multicolumn{11}{|c|}{ Nombre d'articles pour les années 1990, 1991, 1992} \\
\hline \multirow{2}{*}{$\begin{array}{l}\text { Thèmes } \\
\text { Total }\end{array}$} & \multicolumn{2}{|c|}{ IAN } & \multicolumn{2}{|c|}{$V M U$} & \multicolumn{2}{|c|}{ IVGO } & \multicolumn{2}{|c|}{$G P R$} & \multicolumn{2}{|c|}{ TOTAL } \\
\hline & 309 & $\%$ & 255 & $\%$ & 238 & $\%$ & 335 & $\%$ & 1137 & $\%$ total \\
\hline Sous-total: géographie socio-économique & 86 & 27,8 & 60 & 23,5 & 62 & 26,1 & 91 & 27,2 & 319 & 28,1 \\
\hline 1 géographie socio-économique générale & 27 & 8,7 & 15 & 5,9 & 24 & 10,1 & 9 & 2,7 & 75 & 6,6 \\
\hline 2 géographie industrielle & 2 & 0,6 & 12 & 4,7 & 3 & 1,3 & 18 & 5,4 & 35 & 3,1 \\
\hline 3 autres productions matérielles & 5 & 1,6 & 3 & 1,2 & 4 & 1,7 & 3 & 0,9 & 15 & 1,3 \\
\hline 4 transport, commerce, services & 3 & 1,0 & 5 & 2,0 & 3 & 1,3 & 7 & 2,1 & 18 & 1,6 \\
\hline 5 population & 10 & 3,2 & 10 & 3,9 & 5 & 2,1 & 7 & 2,1 & 32 & 2,8 \\
\hline 6 géographie sociale, médicale, des loisirs & 7 & 2,3 & 5 & 2,0 & 8 & 3,4 & 6 & 1,8 & 26 & 2,3 \\
\hline 7 géographie politique, culturelle, ethnique & 6 & 1,9 & 4 & 1,6 & 7 & 2,9 & 1 & 0,3 & 18 & 1,6 \\
\hline 8 ressources naturelles et environnement & 26 & 8,4 & 16 & 6,3 & 18 & 7,6 & 40 & 11,9 & 100 & 8,8 \\
\hline
\end{tabular}




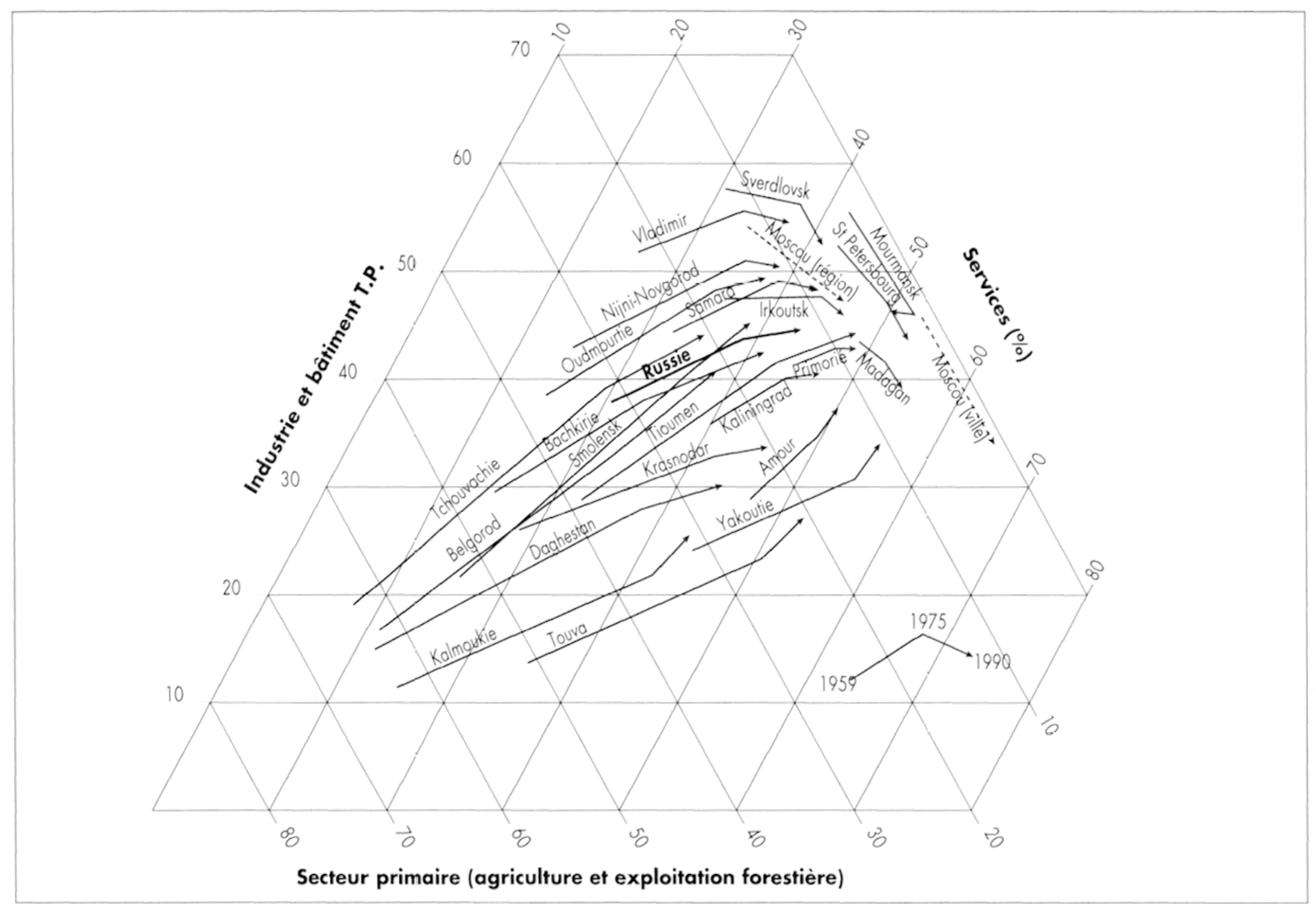

Fig. 1.-Trajets des régions russes dans la répartition des actifs par secteur économique (1970-1992).

On observe un «assèchement» progressif du flux de publications. De 1970-1972 à 1990-1992, la tendance est forte. La relative raréfaction des études industrielles au sens strict semble être une composante d'un phénomène global, propre à l'ensemble de ce qu'on appelait dans le jargon soviétique la «sphère productive» (celle-ci, outre l'industrie, englobait l'agriculture, la construction et le transport de marchandises). Par ailleurs, la géographie de la population a décliné dans les mêmes proportions. On observe par contre une croissance significative des études sociales, ethniques, culturelles et politiques, surtout dans les années 1990, bien qu'en valeur absolue leur nombre soit toujours relativement faible. Le secteur ressources-environnement, qui était très actif au début de la période étudiée, semble devoir le rester aujourd'hui.

L'un de nous (A. Treïvich) a peut-être eu tort d'affirmer, il y a dix ans, qu'une régénération de la géographie industrielle et de la démogéographie était en train de se produire. C'était en partie vrai, et le tableau relatif aux années 1980 à 1982 le confirme, mais il aurait fallu prendre en compte la création de la revue $G P R$, à Irkoutsk. L'augmentation du nombre de publications dans ces domaines au cours des années 1980, notamment sur les thèmes ressources-environnement et plus spécifiquement sur l'industrie, était largement liée à l'apparition de cette revue. Ces articles parus dans GPR sont fort nombreux mais aussi assez spécifiques, parfois très monographiques et souvent consacrés à l'Est du pays (4). Cette revue traite peu de problèmes théoriques et ses problématiques demeurent plus spécialisées.

(4) Concernant l'exploitation forestière autour du Baikal, le bassin de lignite de Kansk-Achinsk ou les problèmes d'utilisation du sol dans la région de Minousisnsk, etc. 
Dans les revues de Russie occidentale, à Moscou et Pétersbourg, la crise est réelle. La géographie industrielle et économique prise comme un tout était le plus important des quatre secteurs de publication au début des années 1970 (27,6\% du total). Au début des années 1990, ce secteur est devenu bon dernier (17,5\%). Ceci est un cas unique: les autres genres connaissent des variations plus mesurées et les évolutions, significatives sur le long terme, sont plus lentes. Ainsi, le secteur population-social-culturel, leader dans les années 1980, est passé de peu derrière le secteur général dans les années 1990 (de 30 à 27\%).

Certaines différences structurelles entre les revues étudiées sont flagrantes. Au cours des années 1980 et 1990, GPR a publié $47 \%$ du total des articles parus sur l'environnement, $43 \%$ de ceux relatifs à l'industrie alors qu'elle ne contribuait que pour $14 \%$ à des articles généraux et théoriques, pour $17 \%$ à la production en géographie de la population, et $9 \%$ seulement aux publications sociopolitiques. Au début des années 1990, GPR publiait même 50\% du total des articles de géographie industrielle! C'est pour cette raison que l'on peut, d'un certain point de vue, parler davantage d'un glissement sibérien, pour la géographie industrielle, que d'une crise globale. La géographie industrielle, lorsqu'elle survit, n'est plus au centre, elle est à l'Est. Elle reprend souvent des problématiques classiques de la géographie soviétique sur la mise en valeur du territoire. C'est ce qui nous a conduit à minimiser son importance dans notre analyse.

$V M U$, de l'université de Moscou, est la publication la plus «industrialiste» après $G P R$, tradition qui remonte sans doute à la période de Baranski et Kolossovski. À l'inverse, $I A N$ et $I V G O$ ont toujours été en tête (y compris devant $G P R$ ) pour la publication des recherches en géographie sociale et de l'environnement. Les deux secteurs pris ensemble faisaient $65 \%$ de la matière parue dans IAN au début des années 1980. IAN a également mené la chasse aux articles de géographie économique et industrielle, espèce qu'elle a pratiquement réussi à éliminer. Dans les années 1990 à 1992, cette revue n'a publié que 10 articles sur ces thèmes, soit 11 à $12 \%$ du total de la matière des $I A N$, dont deux seulement relevant spécifiquement de la géographie industrielle.

L'existence de cette crise fut reconnue dès ses débuts par les instances officielles de la discipline et débattue notam-

\begin{tabular}{|c|c|c|}
\hline \multicolumn{3}{|l|}{$1970,1971,1972$} \\
\hline Sujets & Total & $\%$ \\
\hline Nombre total d'articles & 45 & \\
\hline Nombre total d'items identifiés & 90 & 100 \\
\hline Généralités & 10 & 11,1 \\
\hline Thèmes régionaux & 32 & 35,6 \\
\hline 1 Ensemble de l'URSS & 9 & 28,1 \\
\hline 2 Régions européennes de l'URSS & 7 & 21,9 \\
\hline 3 Régions asiatiques de l'URSS & 7 & 21,9 \\
\hline $4 \quad$ Pays et régions étrangers & 9 & 28,1 \\
\hline Approche sectorielle & 17 & 18,9 \\
\hline 1 Pétrole et énergie & 4 & 23,5 \\
\hline 2 Extraction minière et industrie du bois & 5 & 29,4 \\
\hline 3 Produits de base (chimie, métallurgie) & 4 & 23,5 \\
\hline 4 Construction mécanique & 5 & 29,4 \\
\hline 5 Industrie légère et agro-alimentaire & 3 & 17,6 \\
\hline Thèmes transversaux & 31 & 34,4 \\
\hline 1 Organisation, réseaux, localisation & 8 & 25,8 \\
\hline 2 Technologie & 2 & 6,5 \\
\hline 3 Niveau et processus d'industrialisation & 6 & 19,4 \\
\hline $4 \quad$ Industrie, population et travail & 1 & 3,2 \\
\hline 5 Industrie, environnement et ressources & 12 & 38,7 \\
\hline
\end{tabular}

Tabl. 2.-Analyse thématique et régionale des articles traitant de géographie industrielle.

ment au sein de la branche moscovite de la Société géographique de l'URSS. Bien qu'il ne s'agisse en rien d'un secret d'État, il était pourtant plus facile d'en parler en réunion que d'essayer d'écrire à ce sujet. Au début des années 1980, un éditeur scientifique refusa en effet d'inclure dans une analyse de la bibliographie socio-économique préparée par A. Treïvich quelques pages consacrées au déclin des publications industrielles (5). Il y apparaissait que la géographie industrielle était supplantée par la géographie sociale et de la population, ainsi que par les études sur l'environnement. L'éditeur d'alors n'avait pas voulu accorder d'importance excessive au problème (6).

(5) Les tableaux analytiques associés furent également censurés.

(6) C'est au fond un phénomène assez semblable à ce que Baranski avait dit au début des années 1950 de la géographie humaine: «elle a quasiment disparu, écrasée entre les recherches consacrées à la nature ou à la production matérielle». 


\begin{tabular}{|c|c|c|c|c|}
\hline \multicolumn{5}{|c|}{$1980,1981,1982$} \\
\hline Sujets & total & $\%$ & GPR exclu & $\%$ \\
\hline Nombre total d'articles & 64 & & 39 & \\
\hline Nombre total d'items identifiés & 136 & 100,0 & 78 & 100,0 \\
\hline Généralités & 15 & 11,0 & 12 & 15,4 \\
\hline Thèmes régionaux & 42 & 30,9 & 25 & 32,1 \\
\hline 1 Ensemble de l'URSS & 10 & 23,8 & 10 & 40,0 \\
\hline 2 Régions européennes de l'URSS & 2 & 4,8 & 2 & 8,0 \\
\hline 3 Régions asiatiques de l'URSS & 16 & 38,1 & 2 & 8,0 \\
\hline $4 \quad$ Pays et régions étrangers & 14 & 33,3 & 11 & 44,0 \\
\hline Approche sectorielle & 30 & 22,1 & 12 & 15,4 \\
\hline 1 Pétrole et énergie & 16 & 53,3 & 5 & 41,7 \\
\hline 2 Extraction minière et industrie du bois & 7 & 23,3 & 2 & 16,7 \\
\hline 3 Produits de base (chimie, métallurgie) & 4 & 13,3 & 1 & 8,3 \\
\hline 4 Construction mécanique & 2 & 6,7 & 2 & 16,7 \\
\hline 5 Industrie légère et agro-alimentaire & 8 & 26,7 & 6 & 50,0 \\
\hline Thèmes transversaux & 49 & 36,0 & 29 & 37,2 \\
\hline 1 Organisation, réseaux, localisation & 115 & 30,6 & 12 & 41,4 \\
\hline 2 Technologie & 1 & 2,0 & 0 & 0,0 \\
\hline 3 Niveau et processus d'industrialisation & 3 & 6,1 & 3 & 10,3 \\
\hline $4 \quad$ Industrie, population et travail & 4 & 8,2 & 3 & 10,3 \\
\hline 5 Industrie, environnement et ressources & 20 & 40,8 & 7 & 24,1 \\
\hline \multicolumn{5}{|c|}{$1990,1991,1992$} \\
\hline Sujets & total & $\%$ & GPR exclu & $\%$ \\
\hline Nombre total d'articles & 35 & & 17 & \\
\hline Nombre total d'items identifiés & 54 & 100,0 & 26 & 100,0 \\
\hline Généralités & 6 & 11,1 & 4 & 11,1 \\
\hline Thèmes régionaux & 19 & 35,2 & 11 & 35,2 \\
\hline 1 Ensemble de l'URSS & 4 & 21,1 & 3 & 27,3 \\
\hline 2 Régions européennes de l'URSS & 6 & 31,6 & 4 & 36,4 \\
\hline 3 Régions asiatiques de l'URSS & 5 & 26,3 & 0 & 0,0 \\
\hline $4 \quad$ Pays et régions étrangers & 4 & 21,1 & 4 & 36,4 \\
\hline Approche sectorielle & 16 & 29,6 & 7 & 29,6 \\
\hline 1 Pétrole et énergie & 7 & 43,8 & 1 & 14,3 \\
\hline 2 Extraction minière et industrie du bois & 1 & 6,3 & 0 & 0,0 \\
\hline 3 Produits de base (chimie, métallurgie) & 1 & 6,3 & 1 & 14,3 \\
\hline $4 \quad$ Construction mécanique & 0 & 0,0 & 0 & 0,0 \\
\hline 5 Industrie légère et agro-alimentaire & 7 & 43,8 & 5 & 71,4 \\
\hline Thèmes transversaux & 13 & 24,1 & 4 & 24,1 \\
\hline 1 Organisation, réseaux, localisation & 1 & 7,7 & 1 & 25,0 \\
\hline 2 Technologie & 2 & 15,4 & 2 & 50,0 \\
\hline 3 Niveau et processus d'industrialisation & 0 & 0,0 & 0 & 0,0 \\
\hline $4 \quad$ Industrie, population et travai & 10 & 0,0 & 0 & 0,0 \\
\hline 5 Industrie, environnement et ressources & 10 & 76,9 & 1 & 25,0 \\
\hline
\end{tabular}

méthodes. La forte concurrence d'autres disciplines, l'économie régionale, puis l'économie urbaine, peut sans doute aussi être invoquée.

\section{Les publications de géographie industrielle: structure interne et évolution}

\section{Méthode}

La démarche suivie pour analyser les publications de géographie industrielle repose sur les mêmes principes que ceux appliqués plus haut. Une différence essentielle: un même article a pu être classé dans plusieurs rubriques différentes. Un travail consacré, par exemple, au processus de prise de décision dans l'implantation des raffineries de pétrole en Union soviétique a été défini, pour sa zone de couverture, comme englobant l'ensemble de l'Union, puis du point de vue sectoriel comme relevant du secteur pétrole/énergie, et enfin, pour l'approche, d'un type organisationnel. En principe, le nombre d'items identifiés excède celui des articles de deux fois environ, ce qui semble raisonnable et regonfle quelque peu la faible population traitée (tabl. 2). Néanmoins, il convient de ne pas surinterpréter les résultats présentés ci-dessous: le nombre de cases contenant 0 ou 1 incite à la prudence.

\section{Résultats}

La proportion entre les grands blocs thé-

Cette crise est due à un ensemble de facteurs au sein desquels on peut isoler le manque de données fiables, l'absence de concepts mobilisateurs et novateurs, voire le manque d'enthousiasme des jeunes chercheurs pour une discipline jugée poussiéreuse dans son objet comme ses matiques est plutôt stable, particulièrement le rapport entre items généraux et régionaux. L'absence de changements significatifs à l'intérieur même de chacun des quatre blocs est par contre frappante. Il est assez naturel que la part des articles consacrés à des analyses à l'échelle de l'ex-Union 
soviétique se soit effritée entre le début des années 1980, où elles étaient fort à la mode, et les années 1990 (7). Les études consacrées à des pays étrangers déclinent aussi.

Il est tout à fait remarquable, et même étrange, qu'aucun article significatif n'ait pris comme objet d'étude la Fédération russe, État nouveau et fortement industrialisé, ceci d'autant plus que beaucoup de spécialistes russes des pays étrangers, souvent excellents, ont récemment réorienté leurs recherches en direction de l'espace national. Les nouvelles possibilités d'accès à l'information en Russie même, la demande extérieure (occidentale) d'expertises sur l'exURSS puis la Russie, et aussi la crise de la recherche qui impose un repli (coût d'accès à l'information en provenance de l'étranger, difficulté de financement de missions) seraient pourtant autant de facteurs favorables au développement d'études sur l'industrie nationale. Le vivier de chercheurs travaillant sur l'espace russe s'est donc élargi avec l'apport de géographes dont les thèmes d'étude ont été «rapatriés». La géographie industricllc n'cn a guère profité puisque, conformément à la tendance générale, ces chercheurs ont plutôt investi les champs de la géographie sociale, culturelle et politique, ou tout au plus économique.

L'équilibre relatif observé dans les années 1970 entre les études régionales consacrées aux parties européenne et asiatique de l'URSS se maintient au début des années 1980 (GPR exclu). Mais, au début des années 1990, les publications «européennes» prennent l'avantage; c'est peut-être la traduction du regain d'intérêt signalé plus haut envers les régions anciennement industrialisées. La ventilation par secteur d'activité est assez équilibrée dans les années 1970, sauf peut-être pour le dernier secteur (industrie légère et agro-alimentaire), alors peu prisé. À partir des années 1980 , celui-ci progresse pourtant, même s'il est difficile de parler de boom pour quelque 5-10 articles répartis sur trois ans. On peut l'expliquer autant par la nouvelle actualité du sujet que par l'ouverture des sources. Le même phénomène de croissance se remarque pour le pétrole et l'énergie (catégorie 1), surtout dans les années 1980 avec l'apparition de $G P R$. L'expansion de ces deux secteurs se fait aux dépens des autres, la construction mécanique en particulier (aucun

(7) L'inclusion de la revue sibérienne GPR atténue fortement ces variations. article dans les années 1990!). Enfin, la structure des thèmes transversaux révèle que l'intérêt traditionnel envers les problèmes d'organisation, des réseaux et de localisation (thème 1) que reflète la stabilité du nombre d'items recensés dans les années 1970 puis 1980 ne survit pas à la pérestroïka: un seul article publié sur le sujet dans les années 1990! Le thème 5 (industrie, environnement et ressources), toujours plus important que les autres, connait une croissance portée essentiellement par GPR. Dans les années 1990, ce thème regroupe l'immense majorité $(77 \%)$ des items classés dans les thèmes transversaux.

On pourrait voir un signe de renouveau des problématiques dans la résurrection récente des items technologiques, après leur disparition dans les années 1980. Mais on est trompé par la faiblesse des effectifs traités: il ne s'agit en fait que de deux articles, tous deux parus dans $V M U$ et issus de la désormais défunte chaire d'étude des pays socialistes de l'université de Moscou. L'un d'entre eux, écrit par le professeur $\Lambda$ lissov, est consacré aux biotechnologies en général et le deuxième présente le résultat d'un travail de recherche d'un étudiant (D. Fiodorov) sur le commerce international des brevets. Peut-être pourrait-on y ajouter une seule autre publication, l'article de M. Voïlichnikov sur la localisation des joint-ventures en URSS (paru dans IAN), que nous avons classé dans la catégorie organisation-réseau.

\section{L'entrée dans l'ère postindustrielle, explication du déclin?}

Un genre très particulier de mentalité postindustrielle a animé dans les deux dernières décennies le milieu des sciences sociales, et particulièrement les géographes, à Moscou et Saint-Pétersbourg, les deux capitales intellectuelles du pays. Les thèmes environnementaux et naturels, de géographie sociale ou culturelle attiraient beaucoup plus les étudiants et ont fourni les sujets de nombreuses thèses. Tout ce qui touchait à l'industrie était souvent considéré comme vieillot; et manier tracteurs, productions métallurgiques ou produits chimiques même intellectuellement semblait salissant.

Cette attitude pouvait être confortée par le réel processus de tertiarisation qui a pu être observé au cours des dernières décennies d'existence de l'Union. Dans l'ensemble de 


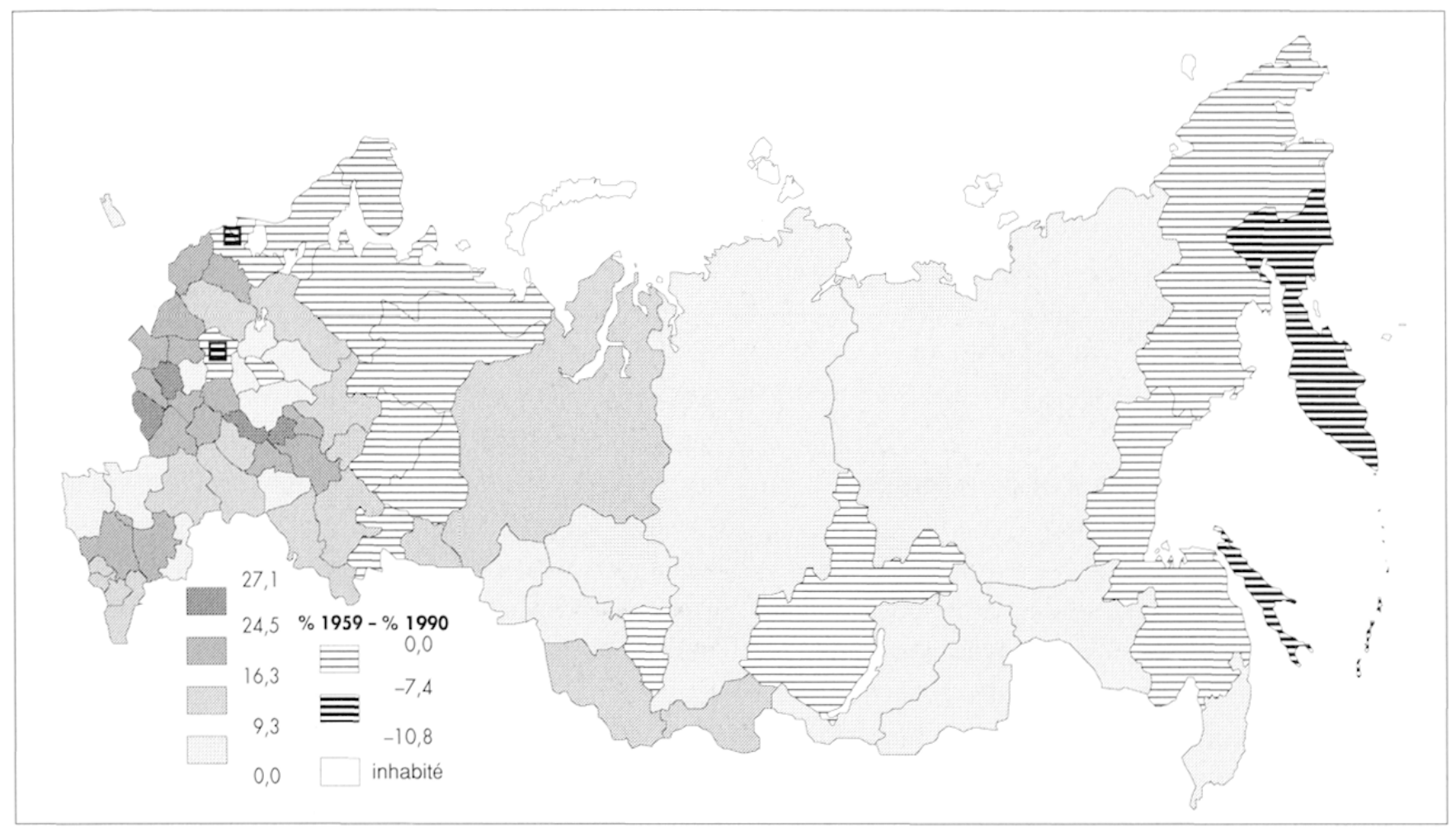

Fig. 2._Les trajectoires de l'emploi industriel dans les régions (1959-1990).

l'URSS, le tertiaire était devenu le premier secteur d'activité au milieu des années 1970 , peu de temps après que l'industrie eut dépassé l'agriculture (Bond, Treïvich, 1993). Néanmoins, il est difficile de parler de désindustrialisation dans certaines républiques (Ukraine, Biélorussie, Lituanie et Arménie), où le secteur secondaire a cru aussi vite que le tertiaire. En Russie même, les trajectoires d'évolution des régions sont très différenciées, assez semblables à celles observées pour les autres membres de l'ex-Union (voir fig. 1). Les régions autonomes, comme le Daghestan, la Kalmoukie ou la république de Touva sont passées directement à une structure postindustrielle, sautant l'étape de l'industrialisation. Les régions plus spécifiquement russes de la périphérie, tout comme quelques régions autonomes incluses dans le couloir de peuplement russe (Oudmourtie, Kalmoukie, Bachkirie) ont suivi l'évolution des régions les plus industrialisées. Et les régions dominantes (grands centres économiques et certaines régions nordiques), sont entrées de plain-pied dans l'économie de services, particulièrement depuis les années 1970. Seul ce groupe, qui avait au départ un secteur primaire inférieur à $20 \%$, connaît un processus de désindustrialisation.
Le processus général de tertiarisation a été finalement assez lent. La part des services (au sens large) dans l'économie n'a pas fondamentalement changé dans les quinze ou vingt dernières années. La tertiarisation a même eu un petit côté illicite, vu la priorité officielle accordée au développement industriel. Les dirigeants étaient particulièrement fiers des résultats de la politique industrielle soviétique, dont la puissance du complexe militaro-industriel était le meilleur témoin (fig. 2). Il est plus que probable que la transition en cours et le processus de privatisation récemment entamé vont puissamment accélérer le processus de désindustrialisation et de montée en puissance des services. Le désengagement des géographes du secteur des études industrielles pourrait s'en trouver accusé.

\section{Une recherche longtemps handicapée}

On a pu observer en Occident la même désaffection à l'égard de l'industrie, dans les années 1970-1980, en pleine crise d'adaptation structurelle de l'appareil productif. Le volumineux Geography: a Modern Synthesis de P. Haggett 
(1972) ne consacre que quelques pages à l'application des modèles de Weber et Lösch à l'industrie. Pourtant, il y a une différence essentielle entre le déclin relatif des problématiques industrielles observé à l'Ouest et la crise de la discipline en Russie. Pourquoi en effet n'y a-t-on pratiquement pas étudié les îlots où se manifestait une dynamique hightech? Pourquoi a-t-on accordé si peu d'attention à la crise profonde des régions anciennement industrialisées, alors qu'en Occident ces deux thèmes ont fait l'objet de recherches attentives et de nombreux débats?

\section{Haute fechnologie, secret ef géographie: un difficile ménage à trois}

La première justification qui vient à l'esprit pour expliquer la quasi-absence de réflexion sur les dynamiques high tech serait l'absence d'une information fiable et accessible. Le fait que la plupart des séries statistiques sur l'industrie collectées par les services de l'ex-Union étaient secrètes n'échappe à personne. Et le peu qui était publié posait bien des problèmes et soulevait des questions cruciales de fiabilité. Même l'exploitation de l'information réputée ouverte pouvait réserver des surprises désagréables: A. Treïvich l'a appris à ses dépens dans les années 1970, lorsqu'il lui fut absolument impossible de publier l'intégralité de ses travaux sur les centrales nucléaires d'Europe de l'Est et d'URSS (A. Treïvich 1979) (8).

Les chercheurs soviétiques pouvaient certes avoir accès aux données estampillées DSP (Dlia Sloujebnogo Polzovania, à usage professionnel) mais, dans ce cas, ils se soumettaient à des restrictions de publication de leurs résultats (D. Eckert, 1993). D'ailleurs, dans les statistiques classées DSP, il y avait aussi des pages blanches. Dans les séries industrielles régionales, les chiffres du secteur des biens d'équipement (construction mécanique et travail des métaux, sidérurgie exclue), étaient absents pour Moscou-ville et pour l'oblast de Sverdlovsk, deux points nodaux du complexe militaroindustriel. Au niveau géographique supérieur, pour les grandes régions économiques correspondantes du Centre et de l'Oural, ils manquaient évidemment aussi, ainsi que

(8) Étude pour laquelle seules des sources publiées et accessibles avaient été utilisées. ceux relatifs à l'ensemble du secteur industriel de l'Union, pour empêcher qu'on puisse les reconstituer.

Il était dans ces conditions bien difficile de travailler. Dans les années 1960, les annuaires statistiques contenaient encore de l'information sur le secteur de l'électronique; mais elle a disparu dans les années 1970! Les recherches ne pouvaient que s'en trouver faussées. L'Institut de géographie de Russie centrale en fit l'amère expérience: une étude entreprise sur la période 1960-1985 aboutissait au paradoxe du déclin de la part des activités high tech dans la plupart des régions, bien que les séries utilisées aient inclus des données sur un grand nombre de branches high tech hors complexe militaire (pétrochimie, biochimie, chimie pharmaceutique, appareillage médical, électrotechnique et appareillage de précision).

Certains pôles d'innovation et de production, situés dans les principales agglomérations du pays, étaient en dehors du champ de la connaissance scientifique. C'était empêcher toute étude sérieuse des fonctions urbaines. L'existence de sites comme Zélénograd (électronique) ou Kaliningrad (recherche et fabrication aérospatiale), tous deux dans l'agglomération de Moscou, était certes connue. Mais la plupart des villes numérotées (les «villes nucléaires» Arzamas-16 et Sverdlovsk-44, par exemple), directement gérées par les ministères soviétiques en charge du complexe militaro-industriel, n'étaient même pas localisées sur les cartes.

On a appris, au fil des découvertes des dernières années, qu'il existait plus de 80 ces villes fermées dans la seule Russie. Très généralement, même dans le milieu des spécialistes soviétiques de l'industrie, l'importance du complexe militaro-industriel a été sous-estimée. Ce n'est qu'aujourd'hui qu'on réévalue les connaissances ou plutôt les hypothèses largement erronées de la période soviétique.

\section{Les lacunes de l'étude des vieilles régions industrielles}

Pour comprendre le déficit de recherche sur les industries et régions industrielles vieillissantes, il faut se souvenir des tabous idéologiques et des particularités économiques du «socialisme réel». Comme il s'agissait d'une économie de pénurie permanente, il n'y avait pas vraiment de crise de la demande, ce qui a longtemps masqué le déclin des vieux foyers industriels. Le discours productiviste officiel, 
avec ses slogans stéréotypés, poussait tous les industriels à produire davantage, plus de charbon, de tissu, de chaussures, de fer et d'acier, de détergents..., quel que soit l'état des usines, même à des conditions de rentabilité incertaines.

Qu'il y ait eu crise structurelle n'était pourtant pas douteux. L'absence de sensibilité des autorités à ce problème a d'ailleurs pu l'aggraver. La priorité accordée à la mise en valeur extensive du territoire orientait les efforts et l'investissement vers les nouveaux espaces "pionniers» de l'industrie. Dans la compétition entre les vieilles régions industrielles et les plus récentes pour l'attribution des investissements distribués par le Centre (9), les premières ont souvent perdu, si bien que le vieillissement de leurs infrastructures s'en est trouvé accéléré et leur capacité à se restructurer amoindrie.

Tout ce qui a pu être dit ou publié sur le thème du déclin des vieilles régions industrielles le fut sur un ton plutôt neutre ou optimiste; ceci alors que, dès les années 1970, le grave mouvement de déprise rurale des Terres non noires attirait l'attention de tous. Il n'était pas de bon ton de s'intéresser aux problèmes des anciens foyers industriels. On peut d'ailleurs trouver dans les publications d'économie régionale de l'époque soviétique quelques jugements étranges. Un article publié dans les années 1970 dans une revue du Conseil d'étude des forces productives, dépendant du Gosplan) critiquait le style de développement spontané et chaotique des vieilles régions industrielles occidentales de l'URSS, opposé au développement planifié, systématique et contrôlé des complexes territoriaux de production de l'Est. Cette approche typique des conceptions du Gosplan n'a heureusement jamais trouvé d'écho très favorable parmi les géographes.

La pérestroïka ayant mis un terme aux projets géants d'aménagement à l'Est de l'URSS (détournement des fleuves sibériens, etc.), on a pu constater à partir de 1985 un regain d'intérêt pour les régions de vieille industrialisa-

(9) Par exemple entre le vieux bassin charbonnier du Donbass et le plus récent Kouzbass, ou entre ces deux régions et les nouvelles zones d'extraction d'hydrocarbures. tion. Peut-être a-t-il fallu que le pays soit plongé dans une crise industrielle gravissime pour mieux appréhender certains problèmes, bien que l'ampleur de la crise puisse, à rebours, par son ubiquité, masquer la réalité des problèmes régionaux et jeter un voile sur la pourtant très réelle crise de certains espaces.

\section{Perspectives}

La situation évidemment mauvaise de la recherche en géographie industrielle doit être évaluée de manière plus approfondie, l'objectif de cet article étant de contribuer au débat. Il est évident que les conditions matérielles dans lesquelles se débattent les chercheurs russes ne sont pas pour rien dans ce déclin, bien que les géographes industriels soient logés à la même enseigne que les autres spécialistes. Les articles parus dans les revues scientifiques signalées n'ont évidemment jamais été rémunérés, mais contribuaient au moins à l'avancement de la carrière de leurs auteurs. Aujourd'hui, la crise du milieu universitaire et de la recherche est telle que les auteurs cherchent à gagner un peu d'argent par tous les moyens, pas forcément en abandonnant toute activité scientifique d'ailleurs, mais en mettant leur compétence et leur capacité de conseil au service des pouvoirs publics russes ou des centres de recherche occidentaux. De surcroît, il est maintenant, à la limite, plus important d'être publié dans des revues étrangères, pour le prestige personnel et pour l'avancement.

Autant la situation des équipes de recherche et des revues scientifiques est franchement mauvaise, voire catastrophique, autant le champ de la recherche est largement ouvert, avec des perspectives très intéressantes. Toute l'analyse de la transition économique et de la restructuration industrielle sera d'un grand intérêt, à la fois dans sa dimension nationale, régionale et internationale (quelle insertion dans le système productif mondial?). Les nouvelles données maintenant accessibles sont un autre facteur d'espérance, ainsi que l'intérêt scientifique international envers le processus de la transition. On ne peut que souhaiter une rénovation de la recherche industrielle russe, à la mesure de l'immensité des transformations du système productif et de son rapport à l'espace géographique. 


\section{Références}

BaranSKI N. (1926). Ekonomitcheskaïa Geografia SSSR (Géographie économique de l'URSS). Moscou.

ECKERT D. (1993). «Données localisées et recherches géographiques en Russie: du problème de la qualité des statistiques russes dans la période de désoviétisation». L'Espace géographique, $\mathrm{n}^{\circ}$ 1, p. 57-62.

HaGgetT P. (1972). Geography: a Modern Synthesis. New York: Harper \& Row.

INTERCARTO (1994) GIS for environmental studies. Actes du colloque, Université Lomonossov, Moscou/Fort Collins, GIS World Books, $143 \mathrm{p}$.

Kolossov V. (1993). «La recherche urbaine en Russie: les nouveaux défis». L'Espace géographique, n 4, p. 289-298.

Kolossovski N. (1969). Teoria ekonomitcheskogo raïonirova- nia (Théorie de la régionalisation économique). Moscou: Mysl.

RADVANYI J. (1982). Le Géant aux paradoxes. Paris: Messidor, $432 \mathrm{p}$.

TARKHOV S., Treivich A. (1992). «Geographical location and diffusion of basic innovations». Geojournal, 26: 3, p. 341348.

TREIVICH A. (1982). «Razvitie promychlennogo proïzvodstva: strouktourno-geografitcheskie svigui i formy territorialnoï organisatsii» (Le développement de la production industrielle: traits structurels géographiques et formes d'organisation territoriale). Itogui Naouki i tekhniki, seria geografia SSSR, tome 16, p. 106-146.

Treivich A. (1979). «Geografitcheskie osobennosti formirovania atomnoï energetiki» (Caractères géographiques de la production d'énergie atomique). Voprosy geografii, vol. 112, p. 75-86.

\title{
Vient de paraître, dans la collection «Espaces modes d'emploi» du GIP RECLUS Statistiques et territoire
}

\section{Joël Charre,}

\author{
professeur à l'université d'Avignon
}

Comprendre les territoires que s'est forgé l'humanité, apprécier les usages qu'elle en fait, en diagnostiquer les contradictions et les tensions, en préciser les dynamiques, tout cela n'est pas d'une mince ambition. C'est celle de la géographie aujourd'hui.

Tout le sens du travail du réseau RECLUS est là: aboutir à des expressions simples des structures et des dynamiques territoriales même complexes, qui soient fondées sur une information suffisante et une analyse stricte supportant épreuve et contreépreuve.

Tel est bien le style habituel de Joël Charre. II travaille sur le fond et sur l'instrument à la fois. II n'aime rien avancer qu'il ne puisse évaluer, mesurer, prouver, comparer. Son nouvel ouvrage est à méditer. II donne les définitions indispensables, les approches utiles, les pièges à éviter, les limites de confiance des méthodes de recherche.

$1995,16,5 \times 24,120$ p., 33 fig., prix: $120 \mathrm{~F}$

ISBN 2-86912-050-2

Diffusion

La Documentation française, 124 rue Henri Barbusse, 93308 Aubervilliers cedex. Tél. (1) 48395600 , Fax (1) 48395601 